\title{
Monazita em veios de quartzo da Serra do Espinhaço Meridional (MG): mineralogia, idades LA-ICP-MS e implicações geológicas
}

\author{
Mario Luiz de Sá Carneiro Chaves ${ }^{1}$, Paulo Roberto Gomes Brandão ${ }^{2}$ \& Bernhard Buhn ${ }^{3}$
}

\begin{abstract}
Resumo Com a descoberta de ocorrências de monazita num ambiente geológico incomum, em veios de quartzo na Serra do Espinhaço Meridional, propôs-se estudar a geologia desses depósitos e seus comportamentos químicos em três localidades (Buenópolis, Olhos d'Água e Diamantina), bem como datações geocronológicas U-Pb por abrasão a laser MC-ICP-MS foram efetuadas em amostras de Diamantina e Olhos d'Água. Em Buenópolis, o mineral aparece como indivíduos geminados, em cascalheiras coluvionares na zona de contato do Grupo Macaúbas com o Supergrupo Espinhaço. Na ocorrência de Olhos d'Água, têm sido registrados grandes cristais em bolsões ovalados métricos, hospedados em metapelitos do Grupo Macaúbas. Em Diamantina, foram estudados cristais milimétricos de um depósito coluvionar do Rio Caeté-Mirim. Certamente, no primeiro e no terceiro casos, tais grãos se originaram dos abundantes veios de quartzo que seccionam as seqüências regionais. Análises com microssonda eletrônica mostraram valores de óxidos em geral semelhantes para os três depósitos, entretanto com certas particularidades. As amostras foram caracterizadas como monazitas-(Ce), localmente ricas em neodímio e, embora em geral não tenham revelado semelhanças absolutas para dois depósitos, Diamantina e Olhos d'Água apresentaram alguns aspectos químicos preferenciais. Os resultados U-Pb indicaram, supondo-se as idades mais antigas ( $491 \mathrm{Ma}$ ) como de cristalização do mineral, uma extensão completa de idades na faixa entre 490 e $440 \mathrm{Ma}$, provavelmente devidas a reaquecimentos tardios. Em termos geotectônicos, tais idades permitem evidenciar as últimas manifestações referentes ao Ciclo Brasiliano na região.
\end{abstract}

Palavras-chave: monazita-(Ce), Serra do Espinhaço, química mineral, geocronologia.

\begin{abstract}
Monazite in quartz-veins from the Southern Espinhaço Range (MG): mineralogy, LAICP-MS ages and geological implications. Due to the discovery of monazite occurrences in an uncommon geologic setting, in quartz veins cutting rocks of the Southern Espinhaço Range, a study was carried out on the geology and mineral chemistry of these deposits in three localities (Buenópolis, Olhos d'Água and Diamantina), as well as to make U-Pb laser ablasion MC-ICP-MS geochronological determinations in the Diamantina and Olhos d'Água samples. In Buenópolis, monazite specimens are common as twin crystals, occurring in colluvial gravel deposits in a contact zone between the Espinhaço Supergroup with the Macaúbas Group. The Olhos d'Água occurrence is in metric oval-shaped pockets hosted in metapelitic rocks of the Macaúbas Group, where large crystals have been discovered. In Diamantina, millimeter-size crystals were studied as broken grains recovered in a colluvial deposit near the Caeté-Mirim river. Certainly in the first and in the third cases, such grains came from the abundant regional quartz veins. Electron microprobe analyses showed similar oxide values for the three deposits, but with some chemical specific aspects. The samples have been identified as monazites-(Ce), locally Nd-rich; the Diamantina and Olhos d'Água deposits seem to have some chemical similarities, although in general there are no absolute resemblance between the two deposits. The U-Pb results showed an older age of $\sim 491$ My that probably indicates the crystallization age of the mineral, and a complete extension between 490-440 My that could mean a later reheating periods. In a geotectonic view, such ages can signify the last events related to the Brasiliano Cycle in the region.
\end{abstract}

Keywords: monazite-(Ce), Espinhaço Range, mineral chemistry, geochronology.

INTRODUÇÃO Importantes mineralizações de monazita no Brasil relacionam-se tanto a depósitos primários como a secundários. Dentre os primários destacam-se em termos genéticos os depósitos em granitos, pegmatitos graníticos, pegmatitos de rochas alcalinas, e de veios de quartzo hidrotermais, enquanto depósitos secundários incluem aluviões e sedimentos de praia, diretamente associados aos primeiros. Depósitos primários de monazita em veios hidrotermais de quartzo são menos comuns em relação aos demais (Spear \& Pyle

1 - Universidade Federal de Minas Gerais, Instituto de Geociências, Centro de Pesquisa Prof. Manoel Teixeira da Costa, Campus da Pampulha, Belo Horizonte (MG), Brasil, e pesquisador CNPq. E-mail: mchaves@ufmg.br

2 - Universidade Federal de Minas Gerais, Escola de Engenharia, Departamento de Engenharia de Minas, Belo Horizonte (MG), Brasil, e pesquisador CNPq. E-mail: pbrandao@demin.ufmg.br

3 - Universidade de Brasília, Instituto de Geociências, Geodinâmicos e Ambientais, Laboratório de Estudos Geocronológicos, Campus Universitário, Brasília (DF), Brasil. E-mail: berbu@unb.br 
2002). Na Serra do Espinhaço (Minas Gerais), há muito tempo é conhecida uma associação do mineral (designado de "rubim" por garimpeiros) com o diamante em sedimentos coluvionares e aluvionares (Gorceix 1885, Derby 1900, Hussak 1917), embora sua fonte primária tenha sido pouco discutida.

O primeiro autor do trabalho tem examinado a assembléia mineralógica dos depósitos diamantíferos secundários dessa região, direcionando também estudos para possíveis áreas onde poderiam estar sua(s) fonte(s) (Chaves 1997). A partir desses estudos, encontraram-se três áreas onde monazita foi identificada, hospedada em veios e/ou bolsões de quartzo encaixados em metassedimentos do Supergrupo Espinhaço e do Grupo Macaúbas. $\mathrm{O}$ estudo da química mineral da monazita em tais ocorrências, bem como suas idades MC-ICP-MS em duas dessas (Diamantina e Olhos d'Água), constituem os objetivos do trabalho. Deve ser ainda destacado o fato que a datação do mineral indica também a idade de cristalização dos veios e, possivelmente, da fase final de deformação associada às unidades geológicas citadas.

A nomenclatura dos minerais portadores de elementos terras raras tem passado por importantes mudanças nas últimas décadas. Para a monazita, por exemplo, eram incluídos em sua fórmula química o tório e o ítrio [(Ce,La,Y,Th)PO4] (eg. Klein \& Hurlbut Jr. 1977), e na atualidade reconhece-se uma distinção entre a monazita comum, ou monazita-(Ce) [de composição ideal $\left.(\mathrm{Ce}, \mathrm{La}, \mathrm{Nd}) \mathrm{PO}_{4}\right]$, de outros três membros mais raros que compõem uma série isomórfica com o predomínio de La, Nd ou Sm: monazita-(La), monazita-(Nd) e monazita-(Sm) (Back \& Mandarino 2008). Estudos recentes têm dado grande atenção ao mineral, por este permitir a um só tempo considerações sobre idade, temperatura e pressão de cristalização de sua rocha encaixante (Boatner 2002, Spear \& Pyle 2002). A presença de monazita em ambientes hidrotermais tem sido destacada em diversos estudos (eg. Bermanec et al. 1988, Chang et al. 1998, Cabella et al. 2001, Spear \& Pyle 2002, Repina 2008), enquanto revisões detalhadas recentes envolvendo datações geocronológicas a partir do mineral são encontradas em Catlos et al. (2002), Harrison et al. (2002) e Vlach (2010), entre outros.

SÍNTESE GEOLÓGICA REGIONAL Todas as ocorrências estudadas se relacionam à Serra do Espinhaço em sua porção meridional (Fig. 1). O Espinhaço como um todo corresponde a um conjunto serrano, que inicia ao sul no Quadrilátero Ferrífero de Minas Gerais, e se estende rumo norte por cerca de $1.200 \mathrm{~km}$ até o extremo norte do Estado da Bahia, com larguras variáveis desde alguns poucos quilômetros até quase 100 $\mathrm{km}$, como no paralelo de Diamantina.

A principal unidade litoestratigráfica que sustenta o espigão de serras é o Supergrupo Espinhaço. Essa unidade foi depositada durante o Paleo-Mesoproterozóico (1,7-1,3? Ga), constituindo quartzitos com intercalações de filitos e metaconglomerados, com raras intrusões de rochas metavulcânicas em sua porção basal (eg., Schöll \& Fogaça 1979, Dossin et al. 1990a,
Machado et al. 1989). Rochas da infraestrutura ocorrem na porção central serrana (região de Gouveia) e na margem leste da mesma. No Espinhaço Meridional, o Supergrupo Espinhaço é sobreposto em suas bordas por metassedimentos do Supergrupo São Francisco, do Neoproterozóico $(0,85-0,6 \mathrm{Ga})$, integrado pelos grupos Macaúbas e Bambuí, o último aflorando exclusivamente a oeste da serra.

Em termos geotectônicos, essas megassequências integram a Faixa de Dobramentos Araçuaí, que margeia o Cráton do São Francisco a leste. A Orogenia Araçuaí desenvolveu-se durante o Ciclo Brasiliano entre 630 e $490 \mathrm{Ma}$ (eg., Uhlein et al. 1986, Pedrosa-Soares et al. 1992, 2001, Uhlein 2001), gerando na Serra do Espinhaço grandes dobramentos de eixos norte-sul com assimetria em direção ao cráton e foliações plano-axiais a tais dobramentos, com mergulhos altos para leste. O metamorfismo superimposto varia do fácies xisto verde a anfibolito, de oeste para leste; processos associados geraram uma miríade de veios de quartzo tardios em ambiente hidrotermal, concordantes ou discordantes da foliação e com forte controle litoestrutural (Chaves et al. 2003, Chaves 2007). Tais veios contêm, localmente, óxidos como hematita, magnetita, rutilo, anatásio, brookita, senaíta e crichtonita, incluindo, em raríssimas ocasiões, euclásio e monazita (Hussak 1917, Chaves \& Karfunkel 1994, Chaves et al. 2006).

DEPÓSITOS ESTUDADOS As principais ocorrências brasileiras de monazita se associam a pegmatitos graníticos da Província Pegmatítica Oriental Brasileira (NE-MG, W-ES e S-BA), bem como a certos plútons graníticos posicionados no Cinturão Atlântico, estes últimos responsáveis pela geração de importantes jazidas detríticas na orla do Espírito Santo e norte do Rio de Janeiro. A partir da descoberta de ocorrências de monazita em ambiente peculiar, em veios de quartzo seccionando litotipos integrantes da Serra do Espinhaço Meridional, propôs-se estudar a geologia dos depósitos e o comportamento químico de amostras selecionadas em três localidades distintas: Buenópolis, Olhos d'Água e Diamantina (Fig. 1).

Em Buenópolis, monazitas aparecem em espécimes de até $3 \mathrm{~cm}$, em geral como indivíduos geminados que produzem interessantes amostras para colecionadores (Fig. 2). Esse depósito, situado na Fazenda Mamonas, constitui cascalheiras coluvionares cobrindo quartzitos grossos ou conglomeráticos do Grupo Macaúbas (Formação Duas Barras), próximo à zona de contato com quartzitos finos e puros do Supergrupo Espinhaço (Formação Galho do Miguel). O mineral foi lavrado intensamente nos anos de 1985-1986, mas desde então os serviços estão paralisados. O estado preservado, sem qualquer arredondamento de tais espécimes, indica uma fonte bastante próxima para a mineralização.

A ocorrência de Olhos d'Água, descoberta recentemente na localidade de Vargem Grande (Chaves et al. 2006, Chaves \& Andrade 2009) em garimpos onde se extrai drusas de quartzo e monocristais de quartzo rutilado para colecionadores, se dá em bolsões ovala- 


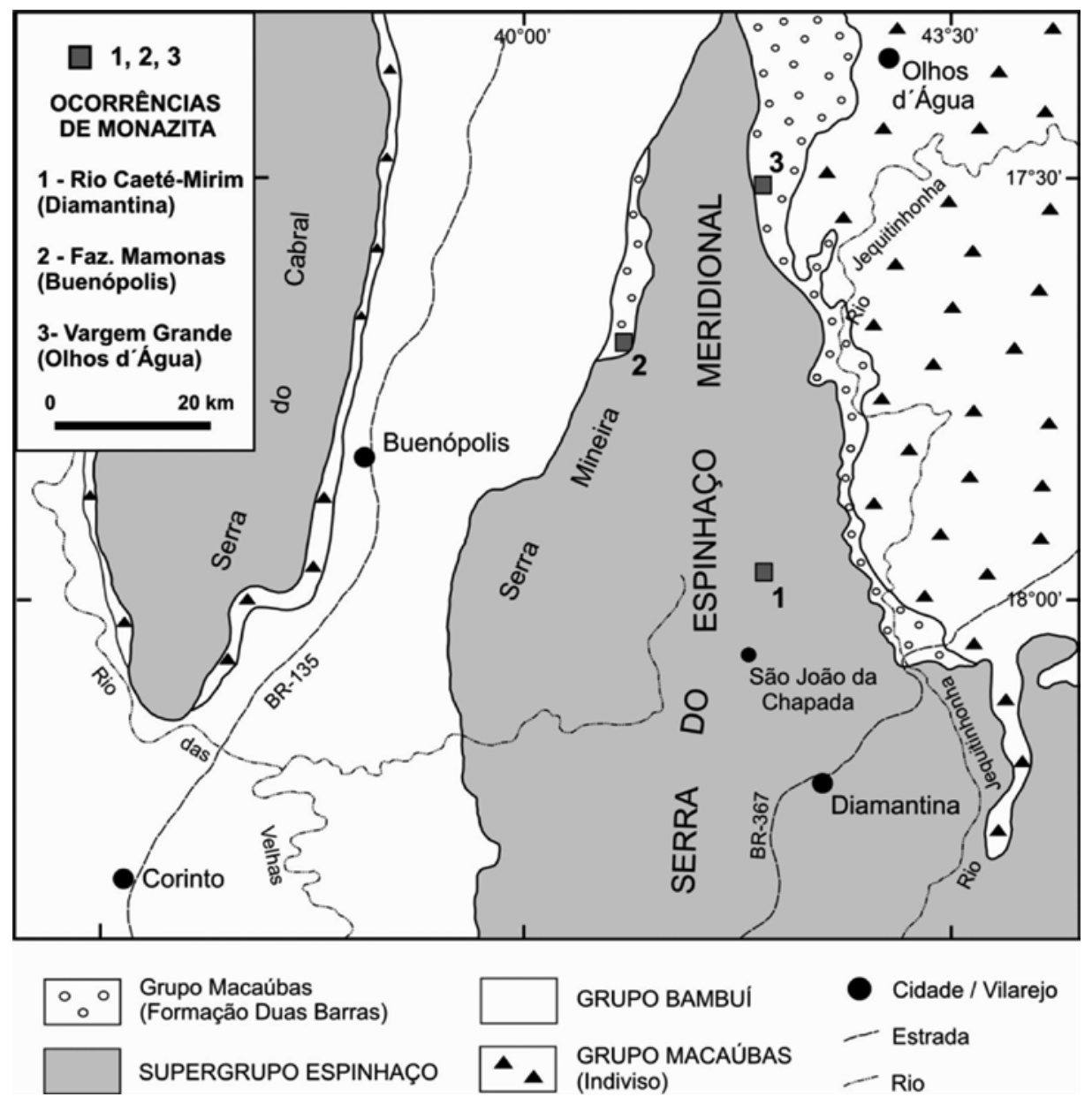

Figura 1 - Geologia da região da Serra do Espinhaço Meridional (parcial e modificada de Heineck et al. 2003), ressaltando-se as três localidades onde foram estudadas amostras de monazita.
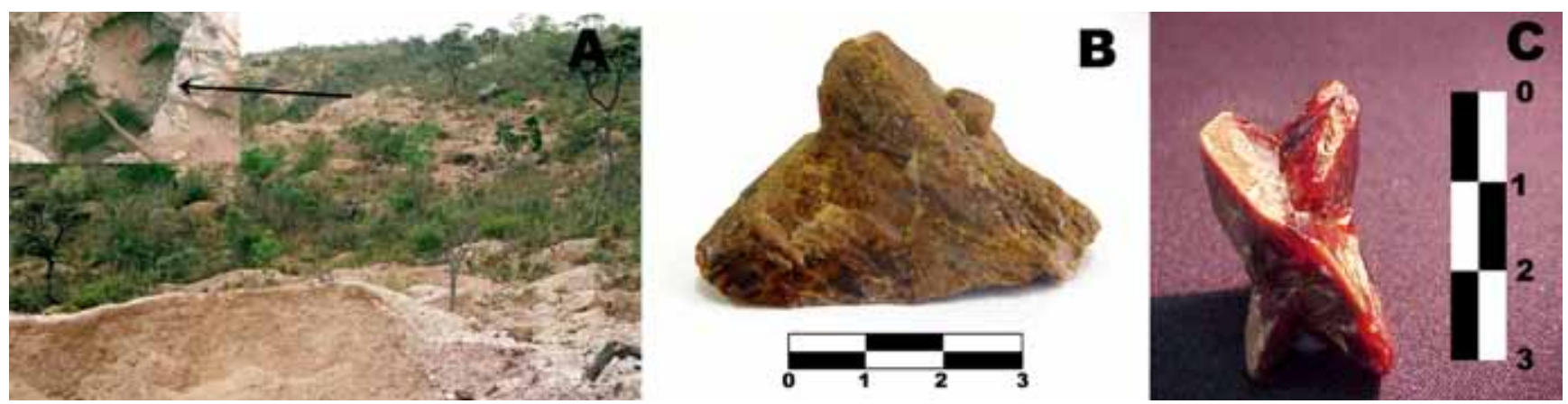

Figura 2 - (A) Visão geral da zona de garimpo de quartzo em drusas e rutilado, com monazita associada, de Vargem Grande (Olhos d'Água), em plena lavra, mostrando em detalhe no alto a esquerda a forma de bolsão ovalado típica dos veios mineralizados; (B) Exemplar de monazita com cerca de 6 cm fotografado nessa área; (C) Exemplar geminado, comum na área da Fazenda Mamonas (Buenópolis), com forte apreciação no ramo do comércio de minerais de coleção.

dos de até $5 \mathrm{~m}$ de diâmetro, inseridos nos metapelitos da base do Grupo Macaúbas (Formação Duas Barras). Nessa localidade, têm sido registrados cristais simples ou geminados com até $8 \mathrm{~cm}$ de comprimento (Fig. 2).
Identificado pelos presentes autores, esse material possui ainda a característica de apresentar inclusões de rutilo, detectado através de difratometria de raios-X.

Foram ainda estudados cristais milimétricos, ge- 
ralmente em grãos angulosos quebrados, de um depósito de rampa coluvionar em direção ao Rio Caeté-Mirim, ao norte de São João da Chapada (Diamantina). De modo diferente dos dois casos anteriores, nessa região afloram exclusivamente litotipos do Supergrupo Espinhaço, e ocorrem tanto cristais perfeitamente euédricos, como também apresentando arredondamento de moderado a médio, denotando diferentes distâncias da área fonte. As rochas encaixantes são representadas por quartzitos e metaconglomerados da Formação Sopa-Brumadinho, cobertos por morros testemunhos de quartzitos finos e puros, pertencentes à Formação Galho do Miguel.

No primeiro e no terceiro casos, os grãos de monazita presentes nos sedimentos superficiais provavelmente originam-se dos abundantes veios de quartzo que ocorrem cortando as seqüências regionais, embora não se tenha observado a presença do mineral in situ.

Química Mineral Estudos prévios, de resultados insatisfatórios, foram efetuados com MEV-EDS em amostras das três localidades citadas (Lab. MEV do Dep. de Engenharia de Minas da UFMG). Novas análises, com microssonda eletrônica (WDS - Lab. de Microanálises, do Depto. de Física da UFMG), tiveram de início fechamentos inferiores ao desejável em termos mineraloquímicos (a maioria menor que 95\%). Para verificar uma possível presença de água na estrutura do mineral, que poderia estar contribuindo nos baixos fechamentos citados, amostras de Buenópolis e Olhos d'Água foram analisadas por termogravimetria TG. Nestas análises, usou-se o instrumento marca Netzsch, modelo STA 409C, em atmosfera de argônio e temperatura máxima de $1.000^{\circ} \mathrm{C}$ (Lab. Magnesita SA). As perdas de massa foram sempre inferiores a $0,05 \%$, de onde se concluiu que tais amostras eram anidras.

As análises químicas com microssonda foram efetuadas em aparelhagem JEOL-JXA-8900RL, equipamento dotado de quatro espectrômetros dispersivos em comprimento de onda, utilizando-se uma tensão de aceleração de $15 \mathrm{kV}$ e corrente de feixe focado de $20 \mathrm{nA}$. Os tempos de contagem foram de 10 s no pico e 10 s no background para $\mathrm{P}, \mathrm{Si}$ e Ca, e de 20 s no pico e 10 s no background para os outros elementos. Entre os padrões: quartzo para $\mathrm{SiO}_{2} ; \mathrm{ThSiO}_{4}$ sintético para $\mathrm{ThO}_{2}$; YAG para $\mathrm{Y}_{2} \mathrm{O}_{3}$; monazita para $\mathrm{P}_{2} \mathrm{O}_{5}, \mathrm{Ce}_{2} \mathrm{O}_{3}, \mathrm{Nd}_{2} \mathrm{O}_{3} \mathrm{e}^{2} \mathrm{La}_{2} \mathrm{O}_{3}$; apatita para $\mathrm{CaO}$; alumina sintética para $\mathrm{Al}_{2} \mathrm{O}_{3}$; $\mathrm{REE} 1$ para $\mathrm{Gd}_{2} \mathrm{O}_{3}$; REE2 para $\mathrm{Sm}_{2} \mathrm{O}_{3}$; e REE3 para $\mathrm{Pr}_{2} \mathrm{O}_{3}$. REE1, REE2 e REE3 são vidros desenvolvidos especialmente para padrões de terras-raras (Drake \& Weill 1972). As seguintes linhas de raios- $X$ foram usadas: $K \alpha$ para Si, P, Ca e Al; L $\alpha$ para Ce, La e Y; L $\beta$ para Nd, Sm, Pr e Gd; e M $\alpha$ para Th.

Tais análises mostraram os teores de óxidos em geral semelhantes para os três depósitos, entretanto com algumas particularidades dignas de nota, assim sumariadas (Tab. 1): (a) $\mathrm{Ce}_{2} \mathrm{O}_{3}$ - predomina em todas as áreas, entretanto em Buenópolis é consideravelmente maior (média de 38,05\%) do que em Olhos d'Água $(29,84 \%)$ e Diamantina (33,73\%); (b) com relação aos outros membros em óxidos da série isomórfica Ce-La-
Nd-Sm, o $\mathrm{La}_{2} \mathrm{O}_{3}$ é quase constante $(12,99-13,33 \%)$, o $\mathrm{Nd}_{2} \mathrm{O}_{3}$ é anomalamente alto em Olhos d'Água (16,26\%), e o $\mathrm{Sm}_{2} \mathrm{O}_{3}$ é um pouco superior em Diamantina e Olhos d'Água (3,17-3,40\%); (c) $\mathrm{ThO}_{2}$ - ocorre em pequena quantidade em Olhos d'Água (1,83\%), apresentando quantidades ínfimas nas demais localidades; (d) $\mathrm{Pr}_{2} \mathrm{O}_{3}$ - é ligeiramente maior em Olhos d'Água $(3,91 \%)$ e o $\mathrm{Y}_{2} \mathrm{O}_{3}$ em Buenópolis $(0,52 \%)$.

Esses dados caracterizam todas as amostras como de monazita-(Ce), localmente ricas em neodímio e, embora não tenham revelado aspectos absolutos de semelhanças entre dois depósitos, o mineral em Diamantina e Olhos d'Água possui certas características químicas em comum. Ressaltam-se ainda os baixos teores gerais de tório, o que contrasta com os valores encontrados em monazita de ambiente granítico-pegmatítico (Marciano 1995) ou de outros locais a nível mundial, onde esse elemento em óxido pode compor até $14 \%$ da estrutura do mineral, motivo pelo qual era inserido em sua fórmula química (Boatner 2002).

Alguns estudos anteriores envolveram a química mineral de monazita em Minas Gerais. Murata et al. (1958) e Marciano (1995) analisaram amostras de pegmatitos graníticos da "Província Pegmatítica Oriental do Brasil", situada a leste da Serra do Espinhaço em Minas Gerais. As médias desses dados constam na tabela 2, juntamente com os espécimes "primários", isto é, cristalizados em rochas graníticas da mesma província (Nalini Jr. 1997), fornecendo-se ainda uma comparação com as médias das análises apresentadas nesse traba1ho. Os dados referentes aos depósitos de Rondônia foram também considerados pertinentes, pois atestam um sistema onde o mineral coexiste nas rochas encaixantes (Souza et al. 2006) e em greisens derivados dessas rochas (Souza et al. 2005). Além disso, uma gênese a partir de greisens já foi sugerida para explicar outros minerais de filiação ácida presentes na Serra do Espinhaço (Chaves et al. 2004).

Do exame da tabela 2, algumas comparações devem ser realçadas em relação ao mineral em depósitos brasileiros (linhas 1-11): (a) $\mathrm{O} \mathrm{SiO}_{2}$ é muito menor nos veios hidrotermais do Espinhaço; (b) Os teores de $\mathrm{ThO}_{2}$ são mais significativos nos sistemas "primários", havendo perda progressiva desse óxido nos "secundários", em ordem decrescente, pegmatitos, greisens e veios hidrotermais; (c) Apesar de não existir grande diferenciação geral entre os valores das terras-raras, existe um certo enriquecimento de $\mathrm{Ce}$ e $\mathrm{Nd}$ no ambiente "secundário", representado pelos veios hidrotermais do Espinhaço; (d) Existe uma presença relevante de $\mathrm{CaO}$ nos sistemas "primários" $(\sim 1 \%)$, valores que se reduzem significativamente nos "secundários"; (e) Comparandose os dados do Espinhaço com os relativos a de outros veios de quartzo hidrotermais (linhas 12-15), onde as principais informações disponíveis referem-se a depósitos europeus e russos, as observações mais importantes dizem respeito à semelhança nos valores de $\mathrm{SiO}_{2} \mathrm{e}$ $\mathrm{ThO}_{2}$; ao $\mathrm{Ce}_{2} \mathrm{O}_{3}$ variável mas em geral menor; ao $\mathrm{La}_{2} \mathrm{O}_{3}$ sempre maior no depósitos do exterior, em geral comparáveis aos dos sistemas granítico-pegmatíticos brasi- 
Tabela 1 - Análises representativas sobre monazita de veios elou coluvionares das áreas: (a) Rio Caeté-Mirim, Diamantina; (b) Vargem Grande, Olhos d'Água e; (c) Fazenda Mamonas, Buenópolis (dados de \% em peso; médias de cinco pontos por grão).

(a) Diamantina

\begin{tabular}{|c|c|c|c|c|c|c|c|c|c|c|c|c|c|}
\hline $\mathbf{N}^{o}$ & $\mathrm{SiO}_{2}$ & $\mathrm{ThO}_{2}$ & $\mathrm{Al}_{2} \mathrm{O}_{3}$ & $\mathrm{La}_{2} \mathrm{O}_{3}$ & $\mathrm{Ce}_{2} \mathrm{O}_{3}$ & $\mathrm{Pr}_{2} \mathrm{O}_{3}$ & $\mathrm{Nd}_{2} \mathrm{O}_{3}$ & $\mathrm{Sm}_{2} \mathrm{O}_{3}$ & $\mathrm{Gd}_{2} \mathrm{O}_{3}$ & $\mathbf{Y}_{2} \mathbf{O}_{3}$ & $\mathrm{CaO}$ & $\mathbf{P}_{2} \mathbf{O}_{5}$ & Tot. \\
\hline 1.1 & 0,01 & 0,27 & 0,59 & 13,81 & 33,70 & 3,38 & 13,81 & 3,02 & 1,92 & 0,42 & 0,04 & 28,69 & 99,24 \\
\hline 1.2 & 0,00 & 0,19 & 0,68 & 13,07 & 33,36 & 3,49 & 14,25 & 3,05 & 2,25 & 0,15 & 0,05 & 28,76 & 99,15 \\
\hline 1.3 & 0,02 & 0,18 & 0,60 & 12,60 & 33,80 & 3,52 & 14,27 & 3,13 & 1,96 & 0,14 & 0,05 & 28,63 & 98,75 \\
\hline 1.4 & 0,02 & 0,07 & 0,68 & 12,87 & 34,08 & 3,52 & 14,21 & 3,34 & 2,05 & 0,11 & 0,05 & 29,22 & 100,11 \\
\hline 1.5 & 0,01 & 0,32 & 0,59 & 12,90 & 33,42 & 3,45 & 14,16 & 3,18 & 2,20 & 0,13 & 0,04 & 28,96 & 99,22 \\
\hline 1.6 & 0,00 & 0,12 & 0,71 & 12,73 & 33,68 & 3,69 & 14,80 & 3,44 & 2,01 & 0,05 & 0,04 & 28,76 & 99,97 \\
\hline 1.7 & 0,00 & 0,16 & 0,70 & 12,96 & 34,22 & 3,48 & 13,83 & 3,03 & 2,01 & 0,16 & 0,05 & 29,28 & 99,71 \\
\hline 1.8 & 0,01 & 0,21 & 0,57 & 13,12 & 33,55 & 3,55 & 14,18 & 3,10 & 1,95 & 0,13 & 0,05 & 29,02 & 99,30 \\
\hline 1.9 & 0,01 & 0,21 & 0,82 & 12,71 & 33,80 & 3,44 & 14,20 & 3,29 & 2,23 & 0,12 & 0,07 & 28,91 & 99,68 \\
\hline Média & $\mathbf{0 , 0 1}$ & 0,19 & 0,66 & 12,97 & 33,73 & 3,50 & 14,19 & 3,17 & 2,06 & 0,16 & 0,05 & 28,91 & \\
\hline
\end{tabular}

(b) Olhos d'Água

\begin{tabular}{|c|c|c|c|c|c|c|c|c|c|c|c|c|c|}
\hline $\mathbf{N}^{0}$ & $\mathrm{SiO}_{2}$ & $\mathrm{ThO}_{2}$ & $\mathrm{Al}_{2} \mathrm{O}_{3}$ & $\mathbf{L a}_{2} \mathbf{O}_{3}$ & $\mathrm{Ce}_{2} \mathrm{O}_{3}$ & $\mathrm{Pr}_{2} \mathrm{O}_{3}$ & $\mathrm{Nd}_{2} \mathrm{O}_{3}$ & $\mathrm{Sm}_{2} \mathrm{O}_{3}$ & $\mathbf{G d}_{2} \mathbf{O}_{3}$ & $\mathbf{Y}_{2} \mathbf{O}_{3}$ & $\mathrm{CaO}$ & $\mathbf{P}_{2} \mathbf{O}_{5}$ & Tot. \\
\hline 3.1 & 0,00 & 0,73 & 1,07 & 13,63 & 30,36 & 4,02 & 16,23 & 3,36 & 2,00 & 0,22 & 0,06 & 27,58 & 99,25 \\
\hline 3.2 & 0,01 & 0,62 & 0,91 & 13,41 & 29,77 & 3,96 & 16,94 & 3,68 & 2,09 & 0,30 & 0,05 & 27,49 & 99,23 \\
\hline 3.3 & 0,01 & 0,88 & 0,96 & 13,88 & 30,92 & 3,98 & 15,72 & 2,71 & 1,67 & 0,26 & 0,07 & 27,68 & 98,72 \\
\hline 3.4 & 0,01 & 2,03 & 0,94 & 13,74 & 30,38 & 3,94 & 16,10 & 3,33 & 1,72 & 0,33 & 0,12 & 27,73 & 100,38 \\
\hline 3.5 & 0,02 & 2,82 & 1,15 & 13,01 & 29,62 & 4,01 & 15,93 & 3,68 & 1,63 & 0,43 & 0,25 & 27,63 & 100,16 \\
\hline 3.6 & 0,01 & 3,87 & 0,98 & 13,08 & 28,98 & 3,71 & 16,18 & 3,55 & 1,97 & 0,71 & 0,52 & 27,28 & 100,82 \\
\hline 3.7 & 0,01 & 1,65 & 0,93 & 12,90 & 29,96 & 4,02 & 16,73 & 3,67 & 1,89 & 0,38 & 0,12 & 28,38 & 100,62 \\
\hline 3.8 & 0,01 & 2,04 & 0,94 & 12,33 & 29,23 & 4,09 & 16,85 & 3,70 & 1,99 & 0,29 & 0,18 & 28,33 & 100,00 \\
\hline 3.9 & 0,00 & 2,20 & 0,93 & 13,89 & 30,07 & 3,76 & 15,57 & 3,01 & 1,70 & 0,37 & 0,17 & 28,08 & 99,74 \\
\hline 3.10 & 0,01 & 1,13 & 1,10 & 13,27 & 29,74 & 3,78 & 16,29 & 3,44 & 1,91 & 0,30 & 0,09 & 28,32 & 99,38 \\
\hline 3.11 & 0,01 & 2,13 & 0,96 & 13,50 & 29,23 & 3,77 & 16,32 & 3,22 & 1,80 & 0,39 & 0,15 & 28,59 & 100,07 \\
\hline Média & 0,01 & 1,83 & 0,99 & 13,33 & 29,84 & 3,91 & 16,26 & 3,40 & 1,85 & 0,36 & 0,16 & 27,92 & \\
\hline
\end{tabular}

(c) Buenópolis

\begin{tabular}{c|c|c|c|c|c|c|c|c|c|c|c|c|c}
\hline $\mathbf{N}^{\mathbf{0}}$ & $\mathbf{S i O}_{2}$ & $\mathbf{T h O}_{2}$ & $\mathbf{A l}_{2} \mathbf{O}_{\mathbf{3}}$ & $\mathbf{L a}_{\mathbf{2}} \mathbf{O}_{3}$ & $\mathbf{C e}_{2} \mathbf{O}_{\mathbf{3}}$ & $\mathbf{P r}_{2} \mathbf{O}_{3}$ & $\mathbf{N d}_{2} \mathbf{O}_{3}$ & $\mathbf{S m}_{2} \mathbf{O}_{3}$ & $\mathbf{G d}_{2} \mathbf{O}_{3}$ & $\mathbf{Y}_{2} \mathbf{O}_{3}$ & $\mathbf{C a O}_{2}$ & $\mathbf{P}_{2} \mathbf{O}_{\mathbf{5}}$ & $\mathbf{T o t . ~}$ \\
\hline $\mathbf{2 . 1}$ & 0,04 & 0,21 & 0,00 & 12,70 & 38,21 & 3,14 & 11,79 & 1,84 & 1,04 & 0,52 & 0,13 & 29,86 & $\mathbf{9 9 , 4 7}$ \\
\hline $\mathbf{2 . 2}$ & 0,03 & 0,55 & 0,01 & 12,95 & 37,49 & 3,21 & 12,24 & 2,18 & 1,19 & 0,42 & 0,15 & 28,82 & $\mathbf{9 9 , 2 3}$ \\
\hline $\mathbf{2 . 3}$ & 0,02 & 0,15 & 0,00 & 13,21 & 37,93 & 3,17 & 12,03 & 2,25 & 1,39 & 0,42 & 0,11 & 29,12 & $\mathbf{9 9 , 8 1}$ \\
\hline $\mathbf{2 . 4}$ & 0,04 & 0,27 & 0,01 & 13,01 & 38,21 & 3,12 & 11,86 & 1,85 & 1,23 & 0,64 & 0,08 & 29,36 & $\mathbf{9 9 , 6 9}$ \\
\hline $\mathbf{2 . 5}$ & 0,02 & 0,34 & 0,01 & 13,21 & 37,58 & 3,14 & 11,87 & 2,23 & 1,55 & 0,51 & 0,11 & 27,38 & $\mathbf{9 7 , 9 4}$ \\
\hline $\mathbf{2 . 6}$ & 0,03 & 0,26 & 0,01 & 13,54 & 38,90 & 3,26 & 11,62 & 1,73 & 1,05 & 0,47 & 0,14 & 29,04 & $\mathbf{1 0 0 , 0 4}$ \\
\hline $\mathbf{2 . 7}$ & 0,01 & 0,10 & 0,00 & 12,89 & 38,40 & 3,37 & 12,18 & 2,05 & 1,26 & 0,68 & 0,08 & 28,92 & $\mathbf{9 9 , 9 5}$ \\
\hline $\mathbf{2 . 8}$ & 0,02 & 0,20 & 0,00 & 13,04 & 37,72 & 3,32 & 12,08 & 2,22 & 1,45 & 0,48 & 0,08 & 29,53 & $\mathbf{1 0 0 , 1 3}$ \\
\hline $\mathbf{2 . 9}$ & 0,01 & 0,36 & 0,00 & 12,91 & 38,11 & 3,25 & 11,61 & 2,11 & 1,18 & 0,72 & 0,12 & 28,93 & $\mathbf{9 9 , 3 1}$ \\
\hline $\mathbf{2 . 1 0}$ & 0,01 & 0,18 & 0,00 & 12,91 & 37,81 & 3,20 & 11,86 & 2,43 & 1,28 & 0,45 & 0,09 & 29,40 & $\mathbf{9 9 , 6 3}$ \\
\hline $\mathbf{2 . 1 1}$ & 0,02 & 0,20 & 0,00 & 13,05 & 38,15 & 3,11 & 12,53 & 2,23 & 1,34 & 0,45 & 0,11 & 28,59 & $\mathbf{9 9 , 7 8}$ \\
\hline Média & $\mathbf{0 , 0 2}$ & $\mathbf{0 , 2 6}$ & $\mathbf{0 , 0 0}$ & $\mathbf{1 3 , 0 4}$ & $\mathbf{3 8 , 0 5}$ & $\mathbf{3 , 2 1}$ & $\mathbf{1 1 , 9 7}$ & $\mathbf{2 , 1 0}$ & $\mathbf{1 , 2 7}$ & $\mathbf{0 , 5 2}$ & $\mathbf{0 , 1 1}$ & $\mathbf{2 8 , 9 9}$ & \\
\hline
\end{tabular}


Tabela 2 - Análises químicas comparativas com monazitas de outras localidades brasileiras e mundiais (dados de \% em peso). Médias aritméticas de: 1,2,3 - Diamantina. Olhos d'Água e Buenópolis (as três, dados do presente trabalho); 4,5,6 - Pegmatitos Grota da Generosa, Morro Escuro e Euxenita (as três de Marciano 1995); 7 - Pegmatito Mar de Espanha (Kato 1958, in Murata et al. 1958); 8,9 - Granitos Urucum e Palmital (Nalini Jr. et al. 1996, Nalini Jr. 1997); 10 - Greisen do depósito Bom Futuro - Rondônia (Souza et al. 2005); 11 - Ortognaisse Jamari - Rondônia (Souza et al. 2006); 12,13,14/15 - Veios hidrotermais em Alinci, ex-Iugoslávia (Bermanec et al. 1988); Liguria Central, Itália (Cabella et al. 2001); e Montes Urais, Rússia, representando (14/15) respectivamente sistemas não alterados e alterados em hidrotermalismo póstumo (Repina 2008). Como os dados constituem médias de muitas amostras, considerou-se desnecessária a apresentação do somatório dos teores em óxidos.

\begin{tabular}{c|c|c|c|c|c|c|c|c|c|c|c|c|c}
\hline & $\mathbf{S i O}_{2}$ & $\mathbf{T h O}_{2}$ & $\mathbf{U}_{\mathbf{3}} \mathbf{O}_{\mathbf{8}}$ & $\mathbf{A l}_{\mathbf{2}} \mathbf{O}_{\mathbf{3}}$ & $\mathbf{C e}_{\mathbf{2}} \mathbf{O}_{\mathbf{3}}$ & $\mathbf{L a}_{\mathbf{2}} \mathbf{O}_{\mathbf{3}}$ & $\mathbf{N d}_{\mathbf{2}} \mathbf{O}_{\mathbf{3}}$ & $\mathbf{S m}_{\mathbf{2}} \mathbf{O}_{\mathbf{3}}$ & $\mathbf{P r}_{\mathbf{2}} \mathbf{O}_{\mathbf{3}}$ & $\mathbf{G d}_{\mathbf{2}} \mathbf{O}_{\mathbf{3}}$ & $\mathbf{Y}_{\mathbf{2}} \mathbf{O}_{\mathbf{3}}$ & $\mathbf{C a O}_{\mathbf{2}}$ & $\mathbf{P}_{\mathbf{2}} \mathbf{O}_{\mathbf{5}}$ \\
\hline $\mathbf{( 1 )}$ & 0,01 & 0,19 & $\mathrm{nd}$ & 0,66 & 33,73 & 12,97 & 14,19 & 3,17 & 3,50 & 2,06 & 0,16 & 0,05 & 28,91 \\
$\mathbf{( 2 )}$ & 0,01 & 1,83 & $\mathrm{nd}$ & 0,99 & 29,84 & 13,33 & 16,26 & 3,40 & 3,91 & 1,85 & 0,36 & 0,16 & 27,92 \\
$\mathbf{( 3 )}$ & 0,02 & 0,26 & $\mathrm{nd}$ & 0,00 & 38,05 & 13,04 & 11,97 & 2,10 & 3,21 & 1,27 & 0,52 & 0,11 & 28,99 \\
$\mathbf{( 4 )}$ & 2,12 & 9,49 & $\mathrm{nd}$ & 0,01 & 33,17 & 13,66 & 9,17 & 1,02 & $\mathrm{nd}$ & $\mathrm{nd}$ & 0,15 & 0,18 & 28,47 \\
$\mathbf{( 5 )}$ & 2,20 & 9,88 & $\mathrm{nd}$ & 0,02 & 34,94 & 15,02 & 6,96 & 0,34 & $\mathrm{nd}$ & $\mathrm{nd}$ & 0,22 & 0,31 & 28,11 \\
$\mathbf{( 6 )}$ & 1,74 & 9,91 & $\mathrm{nd}$ & $\mathrm{nd}$ & 31,24 & 10,07 & 12,12 & 2,58 & $\mathrm{nd}$ & $\mathrm{nd}$ & 1,79 & 0,74 & 29,12 \\
$\mathbf{( 7 )}$ & 1,18 & 5,37 & $\mathrm{nd}$ & 1,08 & 28,43 & 32,44 & $\mathrm{nd}$ & $\mathrm{nd}$ & $\mathrm{nd}$ & $\mathrm{nd}$ & $\mathrm{nd}$ & 0,02 & 28,57 \\
$\mathbf{( 8 )}$ & 0,89 & 7,58 & 0,57 & $\mathrm{nd}$ & 27,99 & 13,48 & 11,94 & 2,03 & 2,99 & $\mathrm{nd}$ & 1,41 & 1,16 & 29,27 \\
$\mathbf{( 9 )}$ & 0,72 & 5,41 & 0,03 & $\mathrm{nd}$ & 29,73 & 15,63 & 12,37 & 1,32 & 2,84 & $\mathrm{nd}$ & 0,13 & 0,83 & 29,39 \\
$\mathbf{( 1 0 )}$ & 0,56 & 2,36 & 0,01 & $\mathrm{nd}$ & 35,71 & 13,77 & 10,85 & 2,39 & 4,20 & 0,91 & $\mathrm{nd}$ & 0,18 & 30,70 \\
$\mathbf{( 1 1 )}$ & 0,32 & 4,69 & 0,62 & $\mathrm{nd}$ & 28,57 & 13,49 & 11,09 & 2,73 & 3,41 & 2,07 & $\mathrm{nd}$ & 1,16 & 30,93 \\
$\mathbf{( 1 2 )}$ & $\mathrm{nd}$ & 3,52 & 0,87 & $\mathrm{nd}$ & 24,57 & 19,62 & 10,89 & 1,78 & 3,85 & 0,30 & 3,22 & $\mathrm{nd}$ & 29,63 \\
$\mathbf{( 1 3 )}$ & 0,06 & 0,44 & $\mathrm{nd}$ & $\mathrm{nd}$ & 31,78 & 13,17 & 15,16 & 2,42 & 2,87 & 1,62 & 1,38 & 0,08 & 30,27 \\
$\mathbf{( 1 4 )}$ & $\mathrm{nd}$ & 3,37 & $\mathrm{nd}$ & $\mathrm{nd}$ & 26,70 & 14,24 & 13,56 & 2,68 & 3,37 & 1,61 & 0,47 & 0,70 & 30,18 \\
$\mathbf{( 1 5 )}$ & $\mathrm{nd}$ & 2,28 & $\mathrm{nd}$ & $\mathrm{nd}$ & 26,98 & 15,22 & 14,15 & 2,82 & 3,55 & 1,26 & 0,48 & 1,46 & 29,41 \\
\hline
\end{tabular}

leiros; e ao $\mathrm{Nd}_{2} \mathrm{O}_{3}$ também semelhante e superior aos de sistemas granítico-pegmatíticos. Embora a depleção em $\mathrm{Si}$, Th e Ca seja característica, esses dados em geral indicam a grande variabilidade composicional da monazita, mesmo em meios semelhantes, e que as diferenças observadas devem representar a constituição química do fluido original, bem como alterações póstumas locais.

Geocronologia Análises isotópicas U-Pb foram obtidas no Laboratório de Geocronologia da Universidade de Brasília para a determinação da idade de cristalização da monazita, em aparelhagem Thermo Neptune, a qual permite detecção de concentrações a nível de ultratraços de isótopos de $\mathrm{Pb}$ e U. As amostras foram analisadas por laser-ablation inductively coupled plasma mass spectrometry (LA-ICP-MS), utilizando os padrões internacionais GJ1 e Temora, em esquema analítico de standard-sample bracketing. Para cada amostra obtevese seis análises pontuais com diâmetros de aproximadamente $100 \mu \mathrm{m}$, totalizando 30 análises, e cada análise consistiu de 40 ciclos de $1 \mathrm{~s}$ cada. A redução dos dados incluiu a consideração dos brancos, o cálculo dos erros 2 sigma do desvio padrão, e os valores theta $(\theta)$ conforme Albarède et al. (2004). A grande maioria dos cristais contém $\mathrm{Pb}$ comum com uma fração de $0,005-0,02$ do $\mathrm{Pb}$ total. Por isso, foi necessária a correção desse elemento utilizando os valores de Stacey \& Kramers (1975). To- dos os cálculos foram feitos com planilha Excel, utilizando os plug-ins de Ludwig (2003) para a preparação das figuras. Outros detalhes sobre o método analítico utilizado são fornecidos em Buhn et al. (2009).

A determinação de idades geocronológicas com tal método foi tentada para amostras das três áreas enfocadas, entretanto somente cristais de Diamantina e Olhos d'Água forneceram resultados analíticos satisfatórios. Todas as amostras possuíam características óticas e morfológicas idênticas, excluindo-se assim populações distintas. Cinco cristais de cada localidade foram analisados, com diâmetros entre 2 e $4 \mathrm{~mm}$. Uma relevante parte das idades obtidas variou entre 470 e $450 \mathrm{Ma}$, ressaltando-se a expressiva distância entre as áreas $(\sim 50 \mathrm{~km})$. Supondo-se as idades mais antigas ( $491 \mathrm{Ma})$ como de cristalização do mineral, identificou-se ainda uma extensão completa de idades na faixa entre 490 e $440 \mathrm{Ma}$ (Fig. 3A-B).

A figura 3-A mostra o diagrama de concórdia para as análises das amostras de Diamantina, e a figura 3-B para as amostras de Olhos d'Água. Os erros são da ordem de 1 sigma erro. As 30 análises de cada amostra definiram uma idade concórdia de $458 \pm 3 \mathrm{Ma}$ (Diamantina), e de $454 \pm 4 \mathrm{Ma}$ (Olhos d'Água). Entretanto, tais análises individualmente encontram-se espalhadas ao longo da linha de concórdia. As idades ${ }^{207} \mathrm{~Pb} / 206 \mathrm{~Pb}$ das análises individuais, distribuídas entre 490 e $440 \mathrm{Ma}$, tem média ponderada de $464 \pm 6,5$ Ma nas amostras de 
Diamantina (Fig. 4-A), e entre 465 e 425 Ma com média ponderada de $444 \pm 4$ Ma nas amostras de Olhos d'Água (Fig. 4-B). De tal modo, é possível que as monazitas tenham cristalizado durante um período mais prolongado, ou então que sofreram reequilíbrios em ambiente de temperatura mais alta, com ou sem perda de $\mathrm{Pb}$. O contexto geológico sugere a segunda interpretação.

A existência de idades tardi-brasilianas ou mesmo pós-brasilianas, evidenciando fases de reaquecimentos póstumos, é comum também nos granitóides e pegmatitos derivados que ocorrem na borda leste da Serra do Espinhaço. Marciano (1985) e Marciano et al. (1993) determinaram idades K/Ar em micas e feldspato de pegmatitos da região de Governador Valadares, variando entre $497 \pm 13 \mathrm{Ma}$ e $502 \pm 31 \mathrm{Ma}$, interpretadas como de cristalização dos corpos. Idades U-Th-Pb algo semelhantes, com $531 \pm 22 \mathrm{Ma}$, foram ainda encontradas em monazita de um pegmatito de Dores de Guanhães (Bilal et al. 1995). A faixa com idades mais jovens obtida nesse mineral também é conhecida em rochas granitóides e pegmatitos da mesma região. Assim,
Bilal et al. (1995) determinaram idades similares (462 $\pm 33 \mathrm{Ma})$ pelo método U-Th-Pb, em monazita do Pegmatito Euxenita (Santa Maria do Itabira), e Herz (1970) identificou idades K/Ar entre 460-450 Ma em micas da suíte granítica "Borrachudos". Por sua vez, Sá (1977) reconheceu essas faixas de idade, através de datações $\mathrm{K} / \mathrm{Ar}$ em muscovita de corpos pegmatíticos de Araçuaí: $490 \pm 12$ Ma no Pegmatito Maxixe, e $467 \pm 18$ Ma no Pegmatito Cachoeira.

Tal coexistência de cristais de monazita com duas idades distintas, em depósitos pegmatíticos cogenéticos em uma mesma faixa orogênica, também foi creditada à atuação de fluidos hidrotermais tardios para a geração mais jovem (Bilal et al. 1995).

\section{IMPLICAÇÕES GEOLÓGICAS E CONSIDERA-} ÇÕES FINAIS A monazita ocorre preferencialmente como um mineral acessório raro em rochas graníticas, bem como em cristais maiores em associação a certos tipos de pegmatitos graníticos.

A presença algo anômala desse mineral na
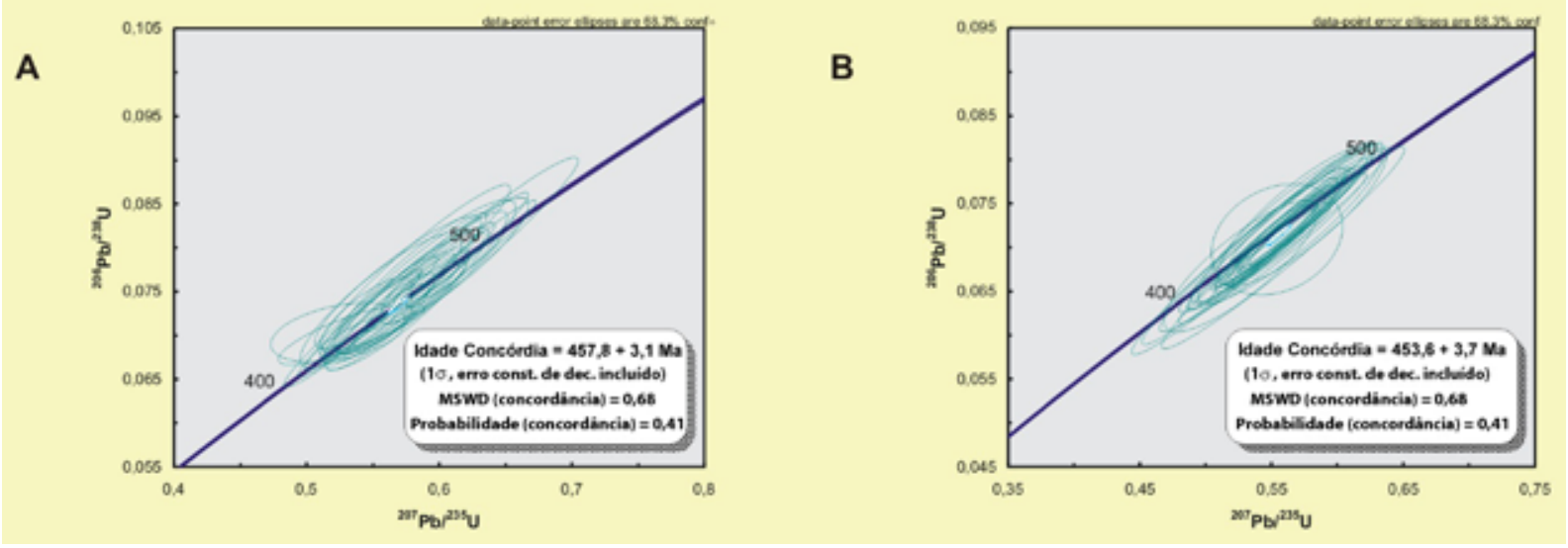

Figura 3 - Diagramas de concórdia das razões isotópicas ${ }^{206} \mathrm{~Pb} /{ }^{238} \mathrm{U}$ versus ${ }^{207} \mathrm{~Pb} /{ }^{235} \mathrm{U}$ para as amostras de monazita em (A) Diamantina e (B) Olhos d'Água (confecções baseadas no programa Isoplot, de Ludwig 2003).
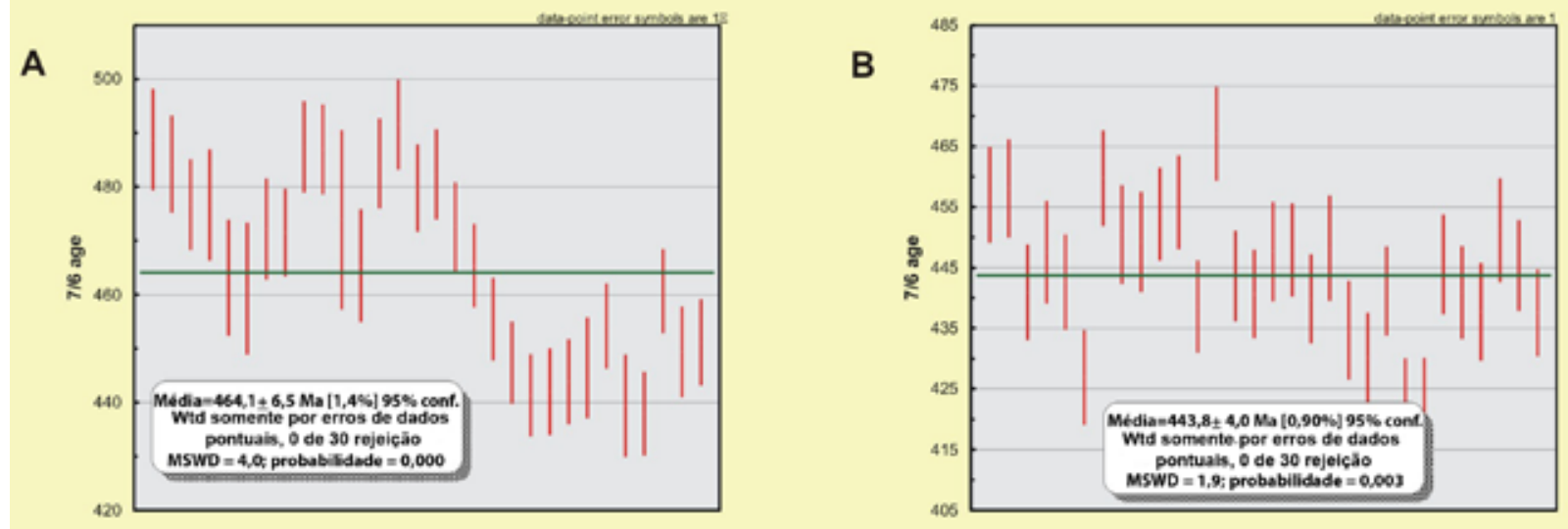

Figura 4 - Diagramas de médias ponderadas para as amostras de monazita em (A) Diamantina e (B) Olhos d'Água (confecções baseadas no programa Isoplot, de Ludwig 2003). 
região de Diamantina foi assinalada desde Gorceix (1885) e Derby (1900). O primeiro, identificou-o em aluviões do Rio Jequitinhonha nas proximidades de Milho Verde, reconhecendo quantidades expressivas de Nd em sua composição. Derby (1900) por sua vez verificou uma abundância extraordinária de grãos frescos de monazita nos concentrados diamantíferos da lavra Cavalo Morto, em Extração, bem como uma outra associação, de monazita com rutilo, nos depósitos de São João da Chapada. Para o primeiro caso, como o mineral era proveniente dos conglomerados proterozóicos da Formação Sopa-Brumadinho, sugeriu-se uma origem em rochas granito-gnáissicas, enquanto para o segundo caso a associação descrita foi relacionada a veios ácidos cortando a sequência regional (Derby 1900).

A ocorrência de monazita em veios de quartzo na região da Serra do Espinhaço, e sua relação com outros minerais típicos de intrusões ácidas, como euclásio, almandina e turmalinas (Chaves 1997, Chaves \& Karfunkel 1994), permite que se suponha a existência de intrusões graníticas sob a serra, de onde proviriam os fluidos mineralizantes. Atualmente, existe um razoável acervo de dados geológicos, tectônicos e geocronológicos a respeito dos eventos magmáticos a nível regional, que podem ser sintetizados e relacionados aos estudos ora apresentados, tendo por base a modelagem regional inicialmente idealizada em Dossin et al. (1990a) (Fig. 5):

1) O Ciclo Orogênico Brasiliano desenvolveuse na maior parte entre idades de 630-490 Ma, com deformação e metamorfismo associado (eg., Uhlein 1991, Uhlein et al. 1986, Noce et al. 2000, Pedrosa-Soares et al. 1992, 2001). Entretanto, eventos póstumos causaram reaquecimentos locais e consequente geração de fluidos mineralizantes, os quais provavelmente ocorreram até quase $100 \mathrm{Ma}$ depois (Dossin et al. 1990b).

2) Esses fluidos foram responsáveis por intenso hidrotermalismo na região de Serra do Espinhaço, formando uma miríade de veios de quartzo que atravessaram a seqüência metassedimentar em período tardi a pós-deformacional. Tais veios se moldaram conforme as estruturas e litologias presentes, e podem apresentar mineralizações em dependência do suprimento local de cátions e/ou complexos metálicos para o abastecimento dos fluidos.

3) $\mathrm{Na}$ margem leste serrana, o Ciclo Brasiliano gerou granitogênese e pegmatogênese em várias etapas (em associação aos granitos das supersuítes "G1" a "G5",

\section{A - NEOPROTEROZÓICO - 900 a 700 Ma}

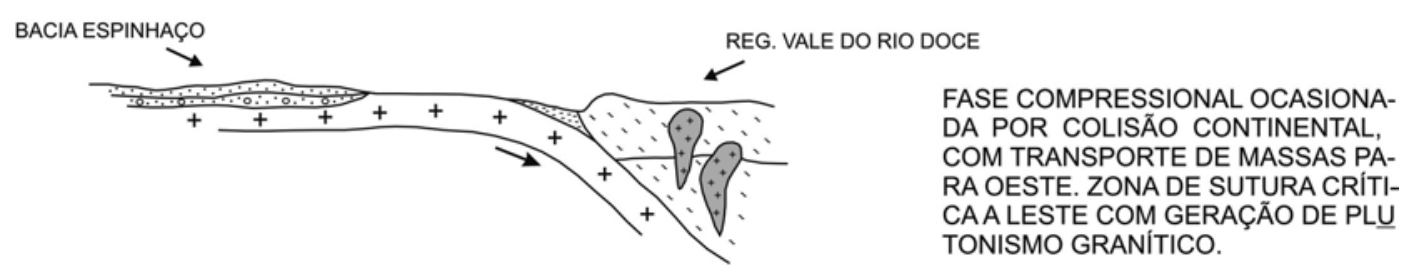

B - NEOPROTEROZÓICO - 700 a 500 Ma

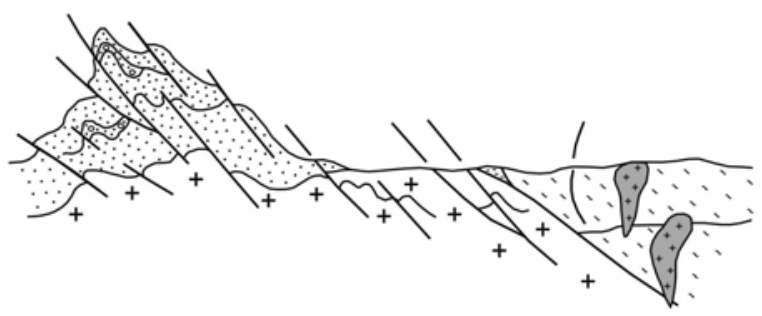

DEFORMAÇÃO COM FRATURAMENTO E MILONITIZAÇÃO DA CROSTA CONTINENTAL. DOBRAMENTOS E METAMORFISMO DE BAIXO GRAU NO SUPERGRUPO ESPINHAÇO.

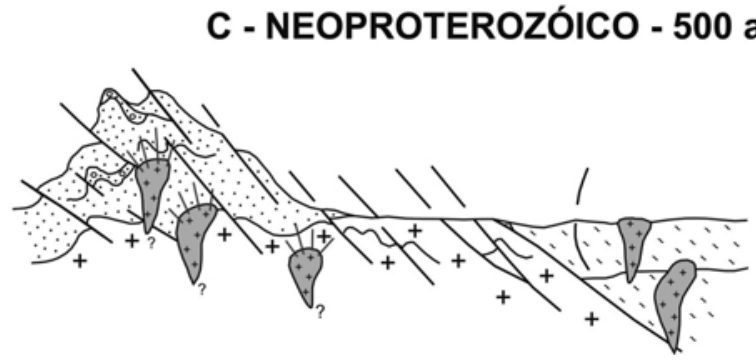

EROSÃO ACENTUADA DA CADEIA DE MONTANHAS. POSSIVEL GERAÇÃO DE PLUTONISMO GRANITICO ANOROGÊNICO COM EMANAÇÕES DE FLUIDOS HIDROTERMAIS.

Figura 5 - Modelo evolutivo da atuação da Orogênese Brasiliana na faixa a leste da Bacia Espinhaço, mostrando as granitogêneses pré a sin-colisional (a e b), e pós-colisional (c), esta última como possível responsável pela geração dos fluidos ácidos que atingiram as rochas metassedimentares da Serra do Espinhaço (adaptada de Dossin et al. 1990a). 
conforme Pedrosa-Soares et al. 2001). Sugere-se aqui, que algumas dessas intrusões, justamente as mais novas ("G4" e "G5") e consideradas anorogênicas (ou pós-colisionais), possam ter alcançado a zona serrana a oeste. Suas idades compreendidas entre 535 a $490 \mathrm{Ma}$ (Pedrosa-Soares et al. 2001), são notavelmente semelhantes às idades mais antigas ora fornecidas no presente estudo. Os fluidos derivados de tais intrusões, provavelmente, foram responsáveis pela deposição dos veios de quartzo com mineralizações ácidas típicas, incluindo a monazita.
Agradecimentos Os trabalhos realizados na região de Olhos d'Água foram parcialmente apoiados pela CPRM (Contrato CPRM-IGC/UFMG 106/PR/06), através do mapeamento geológico da Folha Bocaiúva, sob a responsabilidade do primeiro autor. O geólogo Luiz Garcia efetuou as análises com microssonda eletrônica no Dep. de Física da UFMG. As análises geocronológicas $\mathrm{U}-\mathrm{Pb}$ ocorreram no IG-UnB, sob pedido original do prof. Dr. Nilson F. Botelho. Os dois primeiros autores agradecem ainda ao $\mathrm{CNPq}$, pela Bolsa de Produtividade em Pesquisa.

\section{Referências}

Albarède F., Telouk P., Blichert-Toft J., Boyet M., Agranier A., Nelson B. 2004. Precise and accurate isotopic measurements using multiple-collector ICPMS. Geochimica et Cosmochimica Acta, 68:2725-2744.

Back M.E. \& Mandarino J.A. 2008. Fleischer's Glossary of Mineral Species 2008. Tucson, Mineralogical Record Inc., $345 \mathrm{p}$.

Bermanec V., Tibljas D., Gessner M., Kniewald G. 1988. Monazite in hydrothermal veins from Alinci, Yugoslavia. Mineralogy and Petrology, 38:139-150.

Bilal E., Marciano V.R.P.O., Fuzikawa K., Neves J.M.C., Giret A. 1995. Datação de monazitas do distrito pegmatítico de Santa Maria de Itabira, MG. In: SBG, Simpósio de Geologia de Minas Gerais, 8, Diamantina, Anais, p.46-47.

Boatner L.A. 2002. Synthesis, structure, and properties of monazite, pretulite, and xenotime. Reviews in Mineralogy \& Geochemistry, 48:87-121.

Buhn B., Pimentel M.M., Matteini M., Dantas E.L. 2009. High spatial resolution analysis of $\mathrm{Pb}$ and $\mathrm{U}$ isotopes for geochronology by laser ablation multi-collector inductively coupled plasma mass spectrometry (LA-MC-ICP-MS). Anais da Academia Brasileira de Ciências, 81:99-114.

Cabella R., Lucchetti G., Marescotti P. 2001. Authigenic monazite and xenotime from pelitic metacherts in pumpellyteactinolite facies conditions, Sestri-Voltaggio zone, Central Liguria, Italy. Canadian Mineralogist, 39:717-727.

Catlos E.J., Gilley L.D., Harrison T.M. 2002. Interpretation of monazite ages obtained via in situ analyses. Chemical Geology, 188:193-215.

Chang L.L.Y., Howie R.A., Zussman J. 1998. Rock Forming Minerals, $2^{\text {nd }}$. ed. London, Geological Socity, v.5B, 383 p.

Chaves M.L.S.C. 1997. Geologia e mineralogia do diamante da Serra do Espinhaço em Minas Gerais. São Paulo, Tese de Doutoramento, IG - Universidade de São Paulo, 289 p.

Chaves M.L.S.C. 2007. O megaveio de quartzo da Serra da Catinga (Datas/Gouveia, MG). Geociências, 26:109117.

Chaves M.L.S.C. \& Karfunkel J. 1994. Novas ocorrências de euclásio em Minas Gerais. Boletim IG-USP, Série Científica, 25:53-60.

Chaves M.L.S.C. \& Andrade K.W. 2009. Folha Bocaiúva 1:100.000 Relatório Final. Programa Geologia do Brasil, Convênio CPRM-IGC/UFMG, Brasília, 73 p.

Chaves M.L.S.C., Karfunkel J., Tupinambá M. 2003. Estrutu- ração "em rosário" dos depósitos de quartzo hidrotermal do Morro do Juá (Gouveia, MG). Revista da Escola de Minas, 56:15-20.

Chaves M.L.S.C., Quemeneur J., Tupinambá M., Geraldes M. 2004. Cúpulas graníticas alteradas e mierais aluvionares de origem ácida na Serra do Espinhaço (MG): uma gênese a partir de greisens? In: SBG, Congresso Brasileiro de Geologia, 42, Araxá, Anais, S12-T107 (CD-Rom).

Chaves M.L.S.C., Menezes Filho L.A., Botelho N.F., Brandão P.R.G. 2006. Monazita associada a veios de quartzo hidrotermal na Serra do Espinhaço (MG). In: SBG, Congresso Brasileiro de Geologia, 43, Aracaju, Anais, p.62.

Derby O.A. 1900. Notes on Monazite. American Journal of Science, 10:217-221.

Dossin I.A., Dossin T.M., Chaves M.L.S.C. 1990a. Compartimentação estratigráfica do Supergrupo Espinhaço em Minas Gerais - os grupos Diamantina e Conselheiro Mata. Revista Brasileira de Geociências, 20:178-186.

Dossin T.M., Chaves M.L.S.C., Dossin I.A. 1990b. Mineralizações auríferas associadas às zonas de cisalhamento brasilianas do Espinhaço Meridional (Minas Gerais). Revista de Geologia, 3:19-28.

Drake M.J. \& Weill D.F. 1972. New rare earth elements standards for electron microprobe analysis. Chemical Geology, 10:179-181.

Gorceix H. 1885. Estudo sobre a monazite e a xenotima do Brasil. Annais da Escola de Minas de Ouro Preto, 4:1935.

Harrison T.M., Catlos E.S., Montel J-M. 2002. U-Th-Pb dating of phosphate minerals. Reviews in Mineralogy \& Geochemistry, 48:523-558.

Heineck C.A., Leite C.A.S., Silva M.A., Vieira V.S. 2003. Mapa geológico do Estado de Minas Gerais, Escala 1:1.000.000. Convênio COMIG/CPRM, Belo Horizonte, 1 folha.

Herz N. 1970. Gneissic and igneous rocks of the Quadrilátero Ferrífero, Minas Gerais, Brazil. USGS Professional Papers, 641:1-58.

Hussak E. 1917. Os Satellites do Diamante. Rio de Janeiro, Serviço Geológico e Mineralógico do Brasil, 58 p.

Ludwig K.R. 2003. Isoplot 3.00: A geochronological toolkit for Microsoft Excel. Berkeley Geochronological Center, Special Publication n. 4, 70 p.

Machado N., Schrank A., Abreu F.R., Knauer L.G., AlmeidaAbreu P.A. 1989. Resultados preliminares da geocrono- 
logia U-Pb na Serra do Espinhaço Meridional. In: SBG, Simpósio de Geologia de Minas Gerais, 5, Diamantina, Anais, p.171-174.

Marciano V.R.P.O. 1985. Contribuição ao estudo da mineralogia e geoquímica de pegmatitos da região de Governador Valadares, Minas Gerais. Dissertação de Mestrado, IGc, Universidade de São Paulo, 198 p.

Marciano V.R.P.O. 1995. O Distrito Pegmatítico de Santa Maria de Itabira, MG: mineralogia, gequímica e zoneografia. Tese de Doutorado, IGc, Universidade de São Paulo, 245 p.

Marciano V.R.P.O, Svisero D.P., Neves J.M.C. 1993. Dados geocronológicos de pegmatitos da borda oriental do Cráton do São Francisco. In: SBG, Simpósio do Cráton do São Francisco, 2, Salvador, Anais, p.362-365.

Murata K.J., Dutra C.V., Costa M.T., Branco J.J.R. 1958. Composition of monazites from pegmatites in eastern Minas Gerais, Brazil. Geochimica et Cosmochimica Acta, 16:1-14.

Nalini Jr. H.A. 1997. Caractérisation dês suites magmatiques néoprotérozóiques de la region de Conselheiro Pena et Galiléia (Minas Gerais, Brésil). These de Docteur, Ecole Nationale Superieure dês Mines de Saint Etienne (France), $237 \mathrm{p}$.

Nalini Jr. H.A., Bilal E., Neves J.M.C., Carneiro M.A. 1996. Geoquímica e idades U-Th-Pb de monazitas dos leucogranitos da suíte Urucum, médio Rio Doce, Minas Gerais. In: SBG, Congresso Brasileiro de Geologia, 39, Natal, Anais, p.415-417.

Noce C.M., Macambira M.J.B., Pedrosa-Soares A.C. 2000. Chronology of neoproterozoic-cambrian granitic magmatism in the Araçuaí belt, eastern Brazil, based on single zircon evaporation dating. Revista Brasileira de Geociências, 30:25-29.

Pedrosa-Soares A.C., Noce C.M., Vidal P., Monteiro R.L.B.P., Leonardos O.H. 1992. Toward a new tectonic model for the late proterozoic Araçuaí (SE Brazil) - West Congolian (SW Africa) belt. Journal of South America Earth Sciences, 6:33-47.

Pedrosa-Soares A.C., Noce C.M., Wiedman C.M., Pinto C.P. 2001. The Araçuaí-West Congo orogen in Brazil: an overview of a confined orogen formed during Gondwanaland assembly. Precambrian Research, 110:307-323.
Repina S.A. 2008. Monazite as an indicator of formation conditions of quartz veins at the Zhelannoe deposit, the Subpolar Urals. Geology of Ore Deposits, 50:609-619.

Sá J.H.S. 1977. Pegmatitos litiníferos da região de ItingaAraçuaí, Minas Gerais. Tese de Doutoramento, IGc, Universidade de São Paulo, 112p.

Schöll W.U. \& Fogaça A.C.C. 1979. Estratigrafia da Serra do Espinhaço na região de Diamantina (MG). In: SBG, Simpósio de Geologia de Minas Gerais, 1, Diamantina, Atas, p.55-73.

Souza W.S., Teixeira L.M., Botelho N.F. 2005. Datação U-Th$\mathrm{Pb}$ de monazita hidrotermal e sua aplicação na geocronologia da mineralização de estanho em zonas de greisen do Sistema Granítico Palanqueta, depósito Bom Futuro (RO). Revista Brasileira de Geociências, 35:43-48.

Souza W.S., Teixeira L.M., Dantas E.L., Botelho N.F., Laux J.H. 2006. Idades U-Th-Pb e U-Pb em monazita de ortognaisse do Complexo Jamari, área do depósito de estanho de Bom Futuro (RO). Revista Brasileira de Geociências, 36:71-76.

Spear F.S. \& Pyle J.M. 2002. Apatite, monazite, and xenotime in metamorphic rocks. Reviews in Mineralogy \& Geochemistry, 48:293-335.

Stacey J.S. \& Kramers J.D. 1975. Approximation of terrestrial lead isotope evolution by a two-stage model. Earth and Planetary Science Letters, 26:207-221.

Uhlein A. 1991. Transição cráton - faixa dobrada: exemplo do Cráton do São Francisco e da Faixa Araçuaí (Ciclo Brasiliano) no Estado de Minas Gerais. Tese de Doutoramento, IGc, Universidade de São Paulo, 295 p.

Uhlein A., Dossin I.A., Chaves M.L.S.C. 1986. Contribuição à geologia estrutural e tectônica das rochas proterozóicas e arqueanas da Serra do Espinhaço Meridional. In: SBG, Congresso Brasileiro de Geologia, 34, Goiânia, Anais, 4:1191-1199.

Vlach S.R.F. 2010. Th-U-Pb dating by electron proble microanalysis, Part 1. Monazite: analytical procedures and data treatment. Geologia USP, Série Cientifica, 10:61-85.

Manuscrito ID 16859

submetido em: 18 de fevereiro de 2010 Aceito 22 de novembro de 2010 